Research Paper

\title{
Linearly Patterned Programmed Cell Necrosis Induced by Chronic Hypoxia Plays a Role in Melanoma Angio- genesis
}

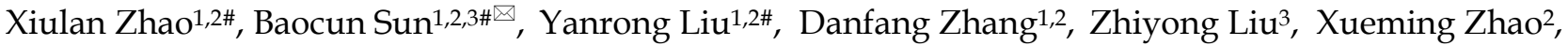 \\ Qiang $\mathrm{Gu}^{1,2}$, Chunrong Han², Xueyi Dong1,2, Na Che ${ }^{1,2}$, Jindan An², Yanjun Zheng2, Tieju Liu ${ }^{1,2}$ \\ 1. Department of Pathology, General Hospital of Tianjin Medical University \\ 2. Department of Pathology, Tianjin Medical University \\ 3. Department of Pathology, Cancer Hospital of Tianjin Medical University \\ \# These authors contributed equally to this work.
}

$\triangle$ Corresponding author: Prof. Sun Baocun, General Hospital and Department of Pathology and Cancer Hospital of Tianjin Medical University, Tianjin, 300052, P. R. China. E-mail: sunbaocun@aliyun.com; fax: (86)-022-83336813.

() Ivyspring International Publisher. Reproduction is permitted for personal, noncommercial use, provided that the article is in whole, unmodified, and properly cited. See http://ivyspring.com/terms for terms and conditions.

Received: 2015.06.10; Accepted: 2015.09.07; Published: 2016.01.01

\begin{abstract}
Background: Highly aggressive tumors are exposed to hypoxia and increased tumor interstitial fluid pressure (IFP) conditions which is resistant to blood supply. Physiological responses of the organism may reduce IFP through induction of orderly cell death.

Specific aims: This study demonstrates that orderly cell death provided spatial structure for early angiogenesis in the hypoxic, high-IFP tumor microenvironment and the participation of linearly patterned programmed cell necrosis (LPPCN) in nascent melanoma angiogenesis.

Methods: Animal model, laser capture microdissection, wound healing and transwell assays, three-dimensional cultures, zymography assays, western-blotting analysis, immunohistochemistry and RT-PCR were performed.

Results: This study demonstrated a special form of cell death occurring in groups of malignant tumor cells which arrayed in lines. Both features of apoptosis and necrosis can be found in this cell death pattern and were termed as LPPCN. Its role as a stimulus of tumor angiogenesis was investigated using human melanoma samples and an animal model. Computer image analysis showed that LPPCN and tumor microvessels had identical spatial distributions. It can be induced by chronic hypoxia, high IFP and subsequent calcium influx. Higher number of tumor associated macrophages (TAM) and VEGF expression were found in the tumor with LPPCN. Based on the tumor-bearing animal model, it was found that block of caspase pathway inhibited LPPCN, microvessel density and vasculogenic mimicry (VM).

Conclusions: LPPCN formation may play an important role in tumor angiogenesis due to stimulation of macrophage infiltration and HIF-1 $\alpha$ regulation, and that inhibition of LPPCN may be a novel therapeutic strategy against tumor angiogenesis and metastasis.
\end{abstract}

Key words: LPPCN; Melanoma; Angiogenesis; VM; IFP; Hypoxia

\section{Introduction}

Melanoma is a prevalent malignancy. Metastasis, in which angiogenesis plays an important role, is the most common cause of death of melanoma patients
(1). Three patterns of tumor microcirculation existed in melanoma including endothelium-dependent vessels (EDVs), VM and mosaic vessels $(2,3)$. The VM 
concept was introduced by Maniotis in 1999 to describe the unique ability of highly aggressive tumor cells to form a capillary-like structure without participation of endothelium cells. VM was related to poor prognosis in cancer patients (4-6).

The growing tumor mass and the high permeability of tumor vessels cause the IFP to elevate (7) which hinders penetration of EDV into tumor tissues and the delivery of drugs(8). Some chemotherapy drugs have been reported to be able to reduce IFP through the induction of cell death $(9,10)$. Vartanian reported that apoptosis occurs before capillary-like structure formation(11). Thus, we speculated that a controlled cell death may be required to reduce the local pressure in the tumor to facilitate angiogenesis.

Cell death can be classified as apoptotic or necrotic, according to classical standards based on cell morphology. Currently, many new terms such as programmed necrosis, necroptosis, and necrosis-like apoptosis were used to describe forms of cell death that do not belong to either classification absolutely (12-15). Our previous work showed a special form of tumor cell death that involved the nucleus condensation, organelle swelling, and membrane rupture, as observed ultrastructurally. Its distribution was similar to EDVs and VM networks and was termed LPPCN to describe the unique distribution patterns of this cell population. We also proved that LPPCN is associated with a short average survival time $(16,17)$.

This study aimed to reveal further the participation of LPPCN in nascent tumor angiogenesis both in vivo and in vitro. It was found that long term mild hypoxia in the tumor tissue can induce the formation of LPPCN but not necrosis or apoptosis with a mild increase of calcium concentration. It was also shown that prevention of LPPCN formation inhibited melanoma angiogenesis and induced enlargement of necrotic areas in tumor tissues and reduced tumor volumes. We speculated that these dead tumor cells provided spatial structure for early angiogenesis in the hypoxic, high-IFP tumor microenvironment.

\section{Materials and methods}

\section{Patient samples}

70 primary melanoma specimens were obtained from patients of Tianjin Cancer Hospital. The diagnoses of these melanoma samples were verified by pathologists.

\section{Cell lines}

The B16F10 mouse melanoma cell line was supplied by the Department of Cell Biology, Huanhu Hospital (Tianjin, China). Trypsin-ethylenediaminetetraacetic acid (trypsin-EDTA) was purchased from Invitrogen (California, USA). The cells were main- tained as a monolayer culture in RPMI 1640 medium supplemented with $10 \%$ fetal bovine serum (FBS; Hyclone) at $37^{\circ} \mathrm{C}$ and $5 \% \mathrm{CO}_{2}$.

\section{Animals and Tumor bearing hind limb ische- mia model}

Please see reference (18) and supplementary material.

\section{Laser capture microdissection (LCM)}

Please see supplementary material.

\section{Vector quantity distribution of LPPCN and blood vessels}

Tissue microarray blocks were constructed from 30 melanoma samples choosing from the 70 samples mentioned above which have LPPCN cells in the tumor. The representative tumor regions were selected and marked on the donor blocks. The selected areas were punched into a cylinder by a $1 \mathrm{~mm}$ diameter hollow needle, and were inserted into an empty paraffin block. Subsequently, these blocks were cut into 100 serial slides ( $4 \mu \mathrm{m}$ thickness), and were prepared for CD31 staining. The immunohistochemistry slide images were recorded using an Olympus BX51 microscope. The microvessels appeared as brown stains in the images, whereas the tumor cells were negatively stained with CD31. The nucleus heterogeneity of LPPCN cells and their affinity to hematoxylin distinguish them from the tumor cells around them. The grayscale threshold value of the tumor tissue was adjusted. The pixel values of LPPCN cells and blood vessels were identified based on the different slides. For each pixel point in the image, the force applied by all other pixels in the image was calculated and summed into a resultant direction vector. About 100 serial sections of the 30 melanoma tissue microarray blocks were divided into sections of 42 small units, such that 30 matrices were obtained. Each matrix was composed of 100 rows and 42 columns. The mean values of the vector matrices of the microvessels and LPPCN cells are listed in the Supplementary Material. Vector mean value curves for the microvessels and LPPCN cells were constructed from the data. Cell pixel was treated as a low gray value images while vascular image is processed for high gray value images. The medical three-dimensional graphics modeling system (3D-DOCTOR 4.0 version) was used for overlay and volume rendering of the tissue images after accurate registration of 100 continuous slides. The pseudo-color was adjusted to distinguish different regions in the images.

\section{Detection of the calcium concentration in the melanoma tissue}

The implanted melanoma tissues, approximately 
$1 \mathrm{~g} /$ specimen, were homogenized in $5 \mathrm{ml} 0.9 \% \mathrm{NaCl}$ solution and were then centrifuged. Calcium concentration in the supernatant material was estimated by the Calcium Detection Kit purchased from abcam company (Cambridge, UK, ab102505) according to the datasheet.

\section{Light microscopic observation and immuno- histochemistry}

The excised sections were pretreated with citrate buffer $(0.01 \mathrm{M}$ citric acid; $\mathrm{pH} 6.0)$ for $10 \mathrm{~min}$ at $95^{\circ} \mathrm{C}$ in a microwave oven. Nonspecific binding sites were blocked by exposure to $10 \%$ normal goat serum for 20 $\min$ at room temperature. The sections were incubated overnight at $4^{\circ} \mathrm{C}$ with a series of primary antibodies (Supplementary Table 1). The staining systems used were PicTure PV6001 and PV6002 (Zhongshan Chemical Co., Beijing, China). The slides were then incubated with 3, 3'-diaminobenzidine (DAB) chromogen for 5-10 min and counterstained with hematoxylin. PBS was used in place of the primary antibodies as the negative control.

The positive staining indices of MMP-2, MMP-9, VEGF, VEGFR1 and VE-Cadherin were assessed according to the method described by Sun (19). The positive rate of pimonidazole staining was quantified by comparing the percentage of positively staining tumor cells in 100 tumor cells in five randomly choosing fields $(100 \times)$ in different regions. The macrophage infiltration was recorded as the average number of the CD68 positive cells in five microscopic fields $(400 \times)$ with the highest macrophage content.

\section{Quantification of microvessel density (MVD), LPPCN cells, necrosis areas and apoptotic cells}

MVD in the endomucin-stained sections were determined according to Weidner (20). LPPCN cells were counted in the H\&E stained sections. The proportion of LPPCN cells in 100 tumor cells in five randomly chosen fields was counted $(400 \times)$. As to the tumor implanted in the mouse skeletal muscle, the tumor areas were defined as tumor areas near skeletal muscle and tumor areas away from skeletal muscle (one 100× high power field from skeletal muscle cells). The average necrotic areas in each tumor and the percentage of Tunel positively staining tumor cells were recorded using the methods provided by Zhang (17). All counts were blindly performed in triplicate.

\section{Electron microscopy}

Tumor specimens of untreated mouse melanoma tissues on 14th day of tumor inoculation were randomly selected and fixed with glutaraldehyde, and then examined by electron microscopy. The LPPCN-containing regions were located by light mi- croscopy. Ultrathin sections (50 nm thickness) were double stained with uranyl acetate and lead citrate, and were then examined under a HITACHI-7500 electron microscope.

\section{Statistical analysis}

All data were evaluated using SPSS13.0 and reported as mean \pm SEM. One-Way ANONA analysis of variance was performed to compare the equality of three or more means while a paired sample t-test was used to determine the differences between the average values of the same measurement made under two different conditions. Wilcoxon rank-sum was used when the population cannot be assumed to be normally distributed. Differences were considered significant at values of $P<0.05$ (marked with *).

\section{Results}

\section{The correlation between LPPCN and tumor blood vessles}

LPPCN cells were often linearly arrayed and interlaced with each other like a network microscopically. Interestingly, this linearly patterned structure was often observed at every 8-10 tumor cell layers and connected with blood vessels. (Figure 1A). Moreover, the LPPCN bands divided the tumor tissue into spherical regions and surrouned the latter, which was consistent with the theory of spherical distribution of tumor blood vessels (21). Counting of the LPPCN cells and MVD in the human melanoma tissue revealed that higher MVD can be found in the patients with LPPCN $(n=38)$ than that without $(n=32)$ (Figure 1B). Besides, LPPCN cell numbers decreased gradually with the implanted melanoma growth time. However, MVD generally increased from the $14^{\text {th }}$ to the $24^{\text {th }}$ day (Figure 1C). A computer image analysis based on three-dimensional reconstruction methods was used to observe the relations between LPPCN and microvessels. The resulting graph showed the coincidence of vector quantity distributions as well as identical spatial distributions between LPPCN cells and microvessels (Figure 1D).

\section{Ultrastructural characteristics of LPPCN}

Figure 2A showed differentially expressed genes of LPPCN and its neighboring tumor cells as well as the ultrastucture morphology of LPPCN cells after LCM (Supplementary Figure 1). IHC detection of HIF-1a, MMP-2, VEGF, VEGFR1 and VE-Cadherin in the untreated melanoma tissues of mice $(n=10)$ was present in Figure 2B. Significantly higher expression of these proteins can be found in the peri-LPPCN regions (left) compared to that without (right) (Figure 2B). 

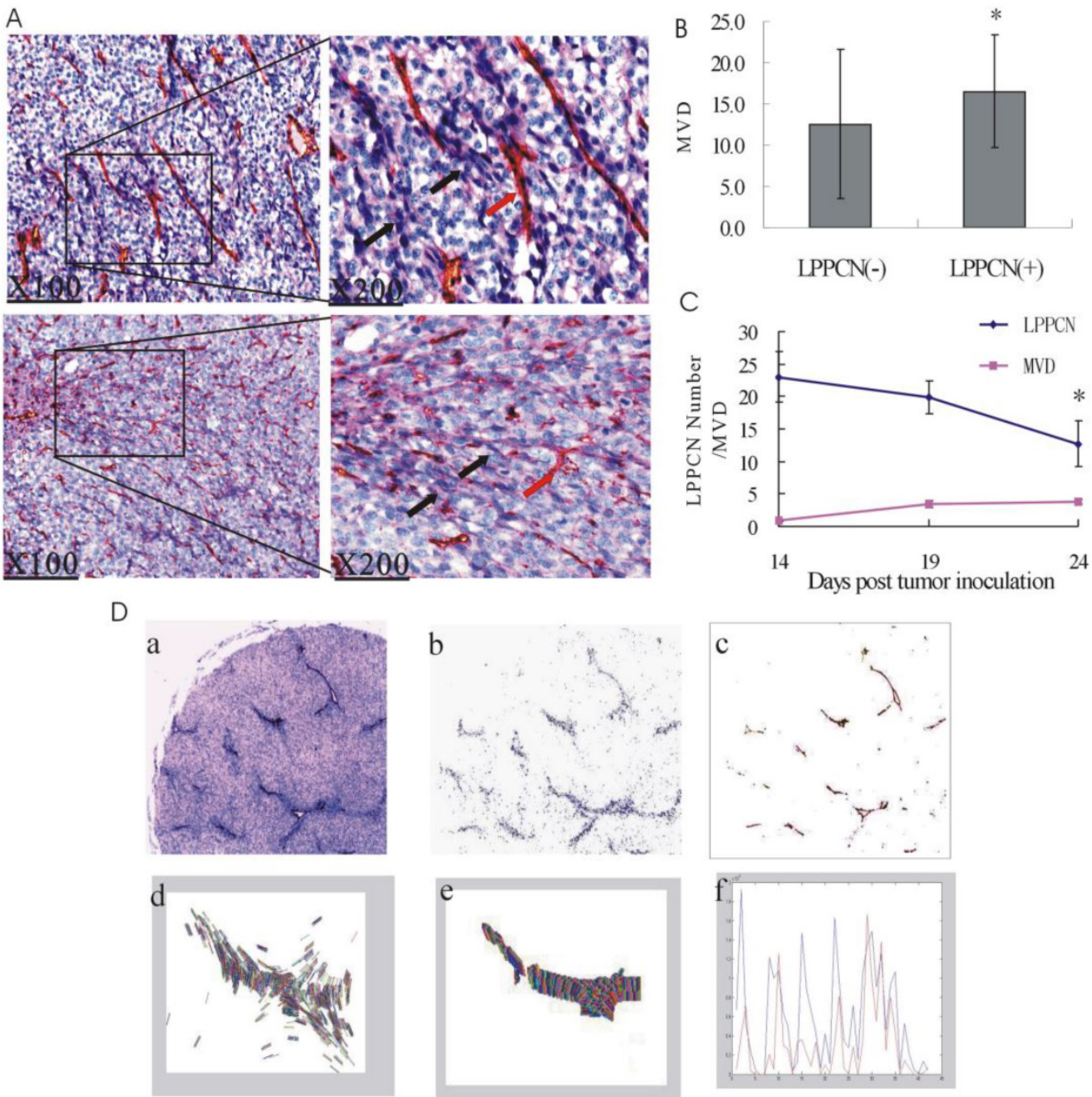

Figure 1. Correlation of LPPCN with tumor blood vessels. (A) LPPCN linked with each other like a network and connected with EDVs. black arrow: LPPCN; red arrow: EDVs. (B) Higher MVD can be found in the human melanoma tissue with LPPCN. (C) LPPCN cell numbers decreased with tumor growth, whereas MVD numbers increased. *P $<0.05$, compared with the $14^{\text {th }}$ day of tumor inoculation. Data was shown as mean \pm SEM ( $n=10$ per group). (D) Computer image analysis of LPPCN and microvessels. (a) CD31 staining of human melanoma tissues showed the identical spatial distribution of LPPCN and blood vessles( $\times 40)$. The pixel data of LPPCN cells (b) and microvessels (c) was extracted from the scanned images of melanoma slides by adjusting the threshold of gray tissue images. The two dimensional vector of LPPCN(d) and microvessels(e) calculated by the way mentioned above in the materials and methods. (f) The curve drawn by mean value of two dimensional vector of LPPCN (blue) and microvessels (red) showed their identical trend of distribution. (g)The three dimensional reconstruction of the scanned images of 100 serial slides of human melanoma tissues (Red arrow: blood vessels; white arrow: LPPCN cells).

Large masses of condensed chromatins can be observed in LPPCN cells ultrastructurally. Mitochondria, endoplasmic reticulum, and other cystic organelles apparently swelled and dilated but lysosomes were not found to be involved. Membranes ruptured and cell junctions such as desmosomes were lost (Figure 2C-d, e). Phagocytosis of LPPCN cells by a macrophage can be observed in Figure 2C-f.

\section{LPPCN is induced by long term chronic mild hypoxia and high IFP in tumors}

More LPPCN cells can be found in the melanoma implanted in skeletal muscle than that implanted in the abdominal cavity (Figure 3A-a, b). Furthermore, there were more LPPCN cells in the tumor areas near skeletal muscles where hypoxia and high IFP were obvious than that in the areas away from the skeletal muscles suggesting that hypoxia and high IFP in the tumor tissue may be inducers of LPPCN (Figure 3A-a, c). The positively staining rate of pimonidazole was much higher in the LPPCN areas and peri-LPPCN areas than that in the no LPPCN areas (Figure 3B). In the hind limb ischemia model, we found that on the early phase of ischemia (the $9^{\text {th }}$ day of tumor inoculation), large areas of necrosis can be induced by severe ischemia while apoptosis can be induced by mild ischemia conditions. However, after long term of chronic mild hypoxia (the $14^{\text {th }}$ day of tumor inoculation), LPPCN number significantly increased compared with the non-ischemia group (Figure 3C). To explore the mechanism behind this phenomenon, we detected the calcium concentration in the tumor tis- 
sues of these three groups which showed a dramatic increase of calcium concentration in the severe ischemia model both on the $9^{\text {th }}$ day and $14^{\text {th }}$ day of tumor inoculation while a mild increase of calcium concentration can be found after long term of chronic mild hypoxia (the $14^{\text {th }}$ day of tumor inoculation) (Table 1).

\section{LPPCN correlated with machrophage infiltra- tion and increases of VEGF expression}

Higher number of TAM and VEGF expression can be found in the human melanoma specimens with LPPCN than that without (Figure 4). Besides, great increase of macrophage infiltration can be found in the mild ischemia group compared with the non- is- chemia group and the severe ischemia group (Figure $5)$.

Table 1. The calcium concentration of tumors between different ischemia model $(\bar{x} \pm s, \mu g / \mu l)$.

\begin{tabular}{llll}
\hline Groups & $\mathrm{n}$ & $9^{\text {th }}$ day & $14^{\text {th }}$ day \\
\hline Control & 10 & $0.370 \pm 0.124$ & $0.403 \pm 0.123$ \\
Severe & 10 & $1.451 \pm 0.360^{*}$ & $1.560 \pm 0.389^{* *}$ \\
Mild & 10 & $0.516 \pm 0.100$ & $0.921 \pm 0.267^{* *}$ \\
F & & 66.637 & 45.424 \\
$P$ & & 0.000 & 0.000 \\
\hline
\end{tabular}

* Significantly different compared with the untreated implanted tumor (control) on the $9^{\text {th }}$ day of tumor inoculation; **Significantly different compared with the control group on the $14^{\text {th }}$ day of tumor growth.
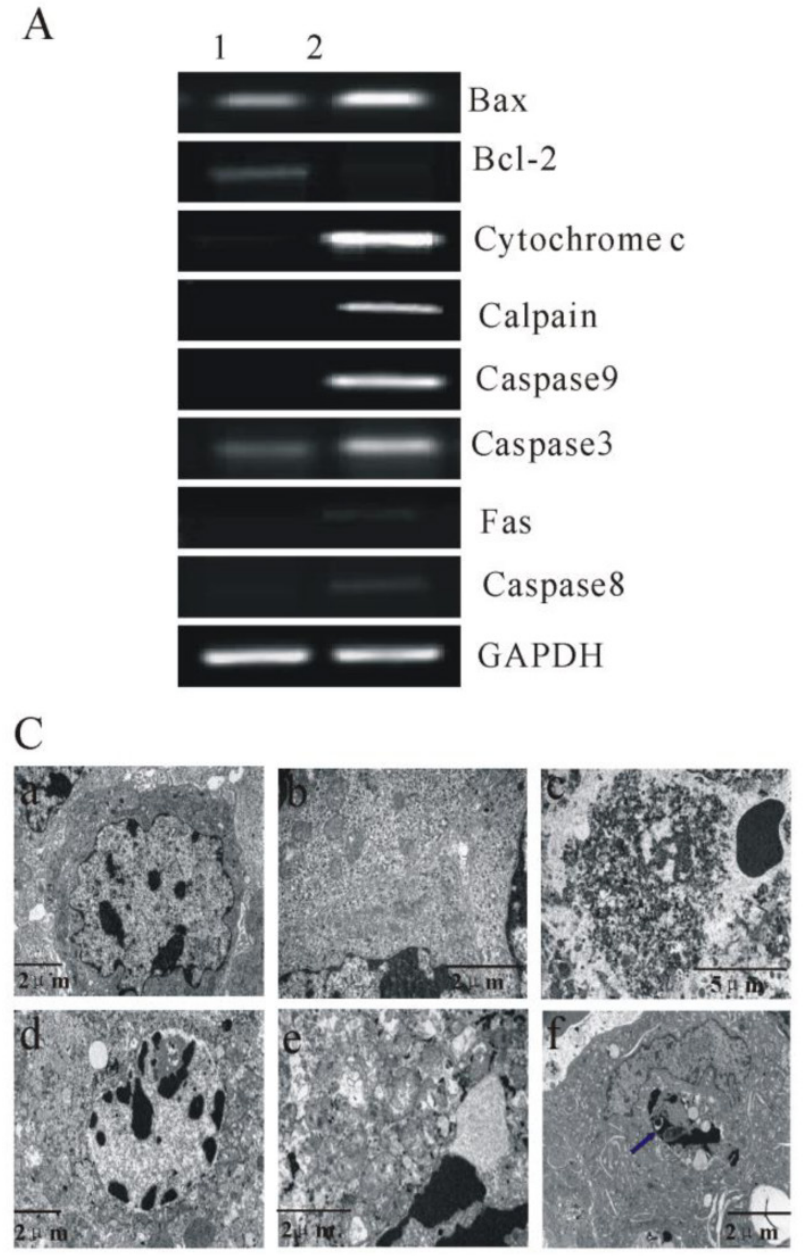

B
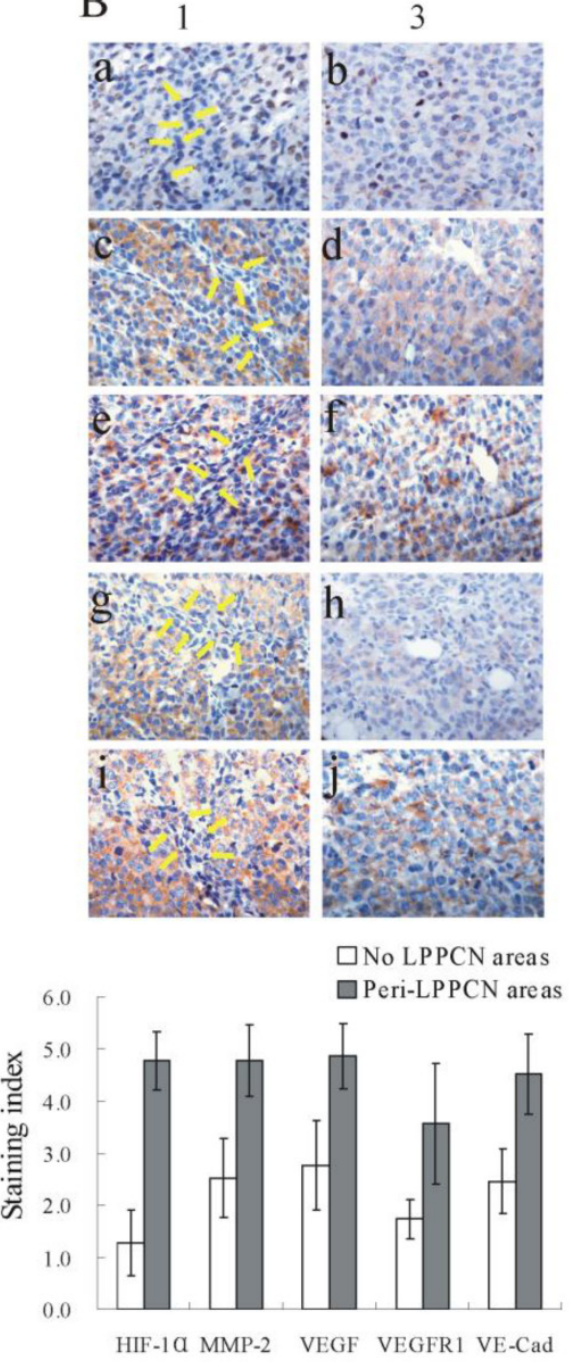

Figure 2. Differentially expressed genes of LPPCN and its neighboring tumor cells as well as the ultrastucture morphology of LPPCN cells. (A) RT-PCR results after LCM. Lane 1: peri-LPPCN regions; lane 2: LPPCN areas. Representative images from three independent experiments are shown. (B) IHC detection of HIF-1a (a,b), MMP-2 (c,d), VEGF (e,f), VEGFR 1 (g,h) and VE-Cadherin(i,j) in the untreated melanoma tissues of mice( $n=10)$. Significantly higher expression of these proteins can be found in the peri-LPPCN regions (left) compared to that without(right). (C) a and b showed the normal melanoma cells while d and e represented LPPCN cells with lager masses of condensed chromatin(d) and extensive vacuolization and swollen organelles (e). Ultrastructure features of classic necrosis were shown in(c) with extensive DNA hydrolysis, vacuolation of the endoplasmic reticulum, organelle breakdown, and cell lyses. (f) LPPCN cell was taken up by a macrophage in which LPPCN particles with condensed chromatin, swollen cytoplasm and loss of membrane integrity (blue arrowhead) were engulfed. No membrane-bound apoptotic bodies can be detected. N: the nucleus of the macrophage. 

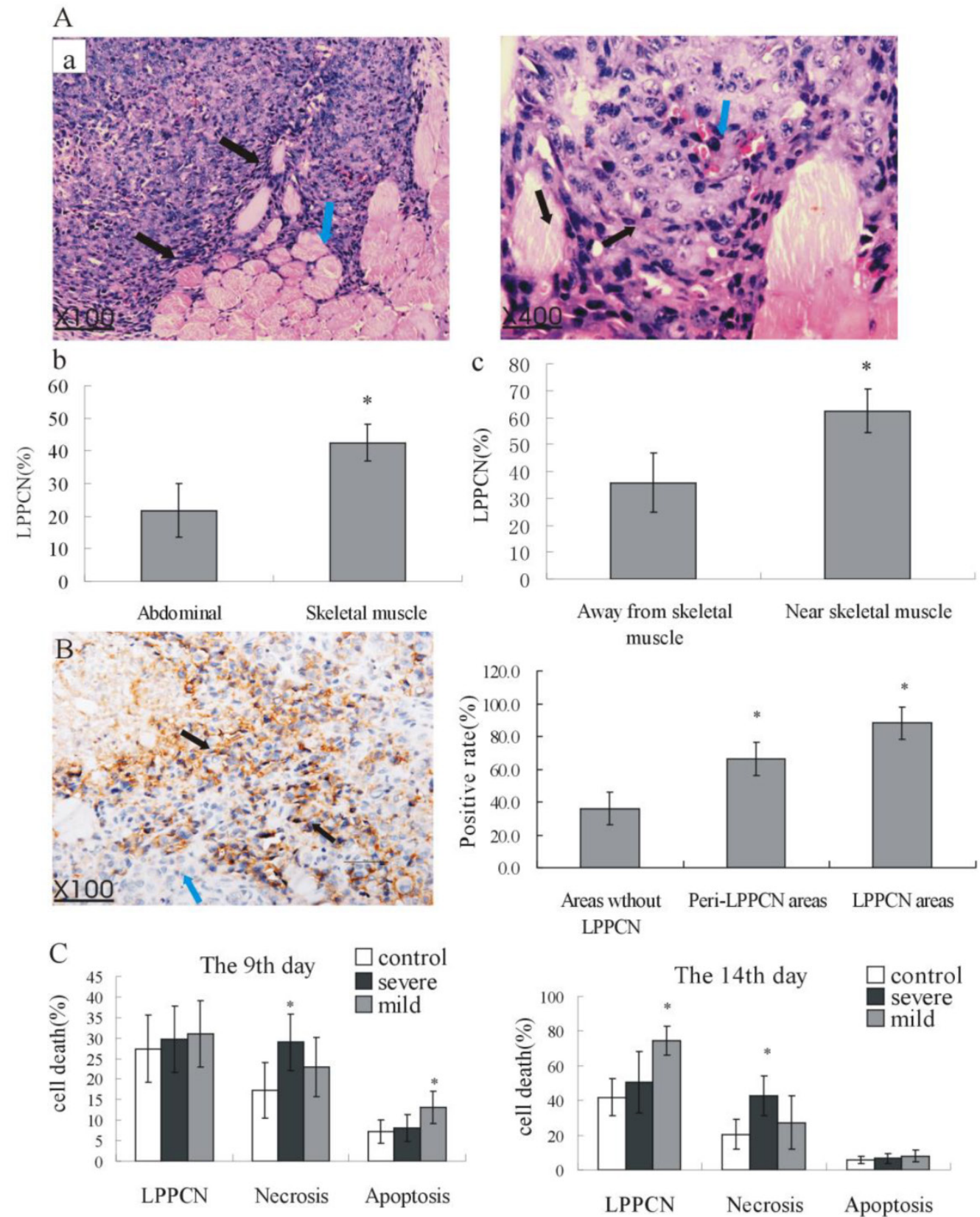

Figure 3. LPPCN can be induced by high IFP and hypoxia. (A) (a, c) LPPCN cells (black arrow) were always located surrounding the skeletal muscles tissue (blue arrow) (b) More LPPCN cells existed in tumors implanted in the mouse skeletal muscle.(B) Higher positively staining of pimonidazole in the LPPCN regions (black arrowheads) compared with that in the peri-LPPCN areas (blue arrowheads) and areas without LPPCN $(100 \times)$ ). $P<0.05$, compared to the areas without LPPCN. (C) Different tumor cell death type in tumor bearing hind limb ischemic model. $* P<0.05$, compared to the control group.

\section{Caspase pathway blockage inhibited melano- ma growth via the inhibition of LPPCN for- mation and lower expression of MMP-2, VEGF and VEGFR 1}

Tumor volumes in mice in the DEVD and LEHD groups were significantly smaller than those in the DMSO control group on the $14^{\text {th }}, 19^{\text {th }}$ and $24^{\text {th }}$ day of tumor inoculation $(P<0.05)$ (Figure 6A). Differences in the count of LPPCN cells suggested that blocking the caspase pathway could reduce LPPCN. This was especially apparent in the DEVD and LEHD groups in which MVD and VM number also decreased and the necrotic areas were enlarged (Figure 6B, C, D, E). TAM number decreased after treatment with DEVD or LEHD while little expression of HIF-1a can be observed in these two groups (Figure 6F, G). No significant differences were found in the expression VE-Cadherin in different treatment groups. 

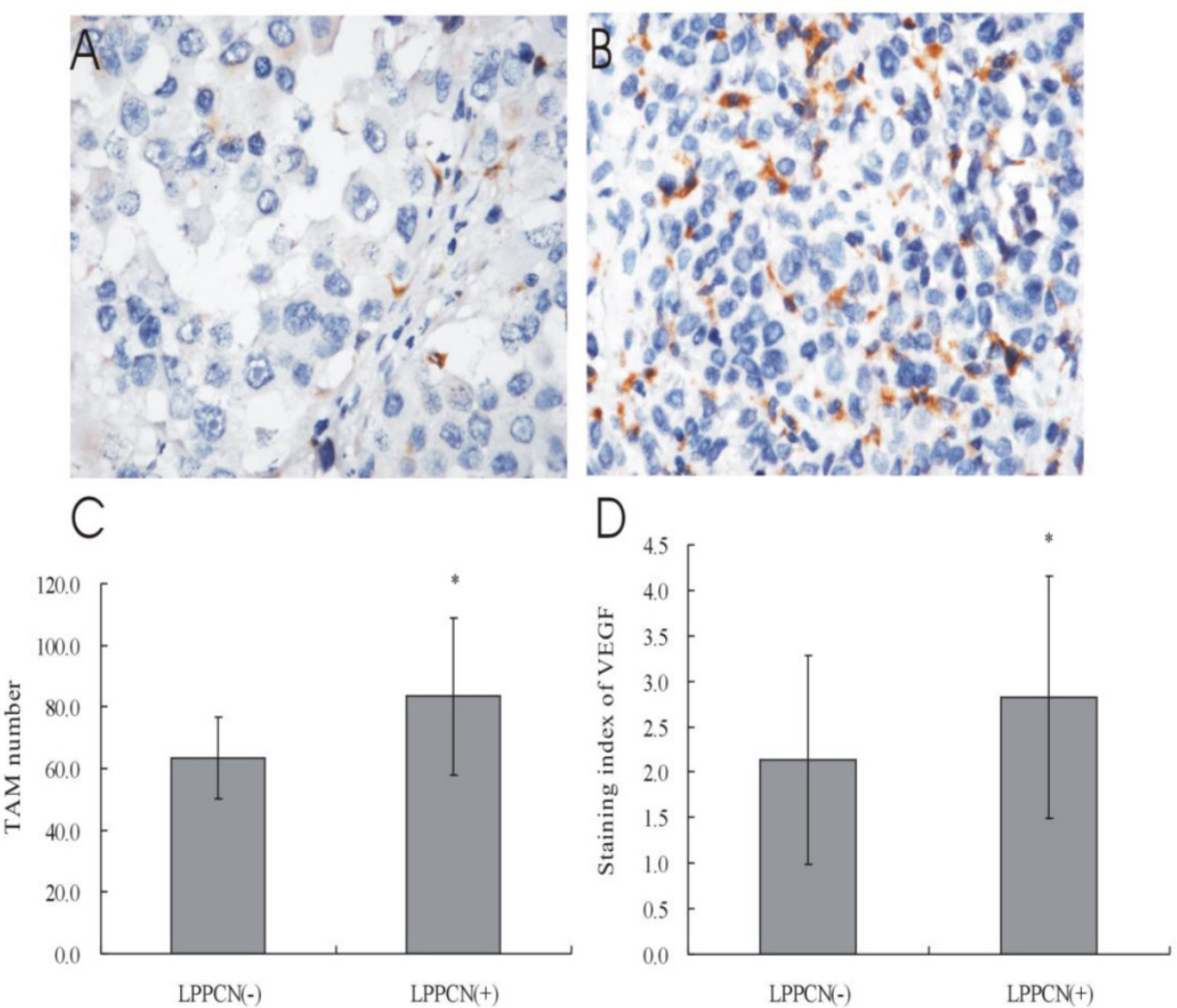

Figure 4. Correlation of LPPCN with machrophage infiltration and VEGF expression. (A, B, C) More CD68 positively staining cells can be detected in the human melanoma tissues with LPPCN and the TAM can be found around LPPCN cells. (D) Higher staining indices of VEGF in macrophages were found in the LPPCN (+) group compared with the LPPCN (-) group (400x).
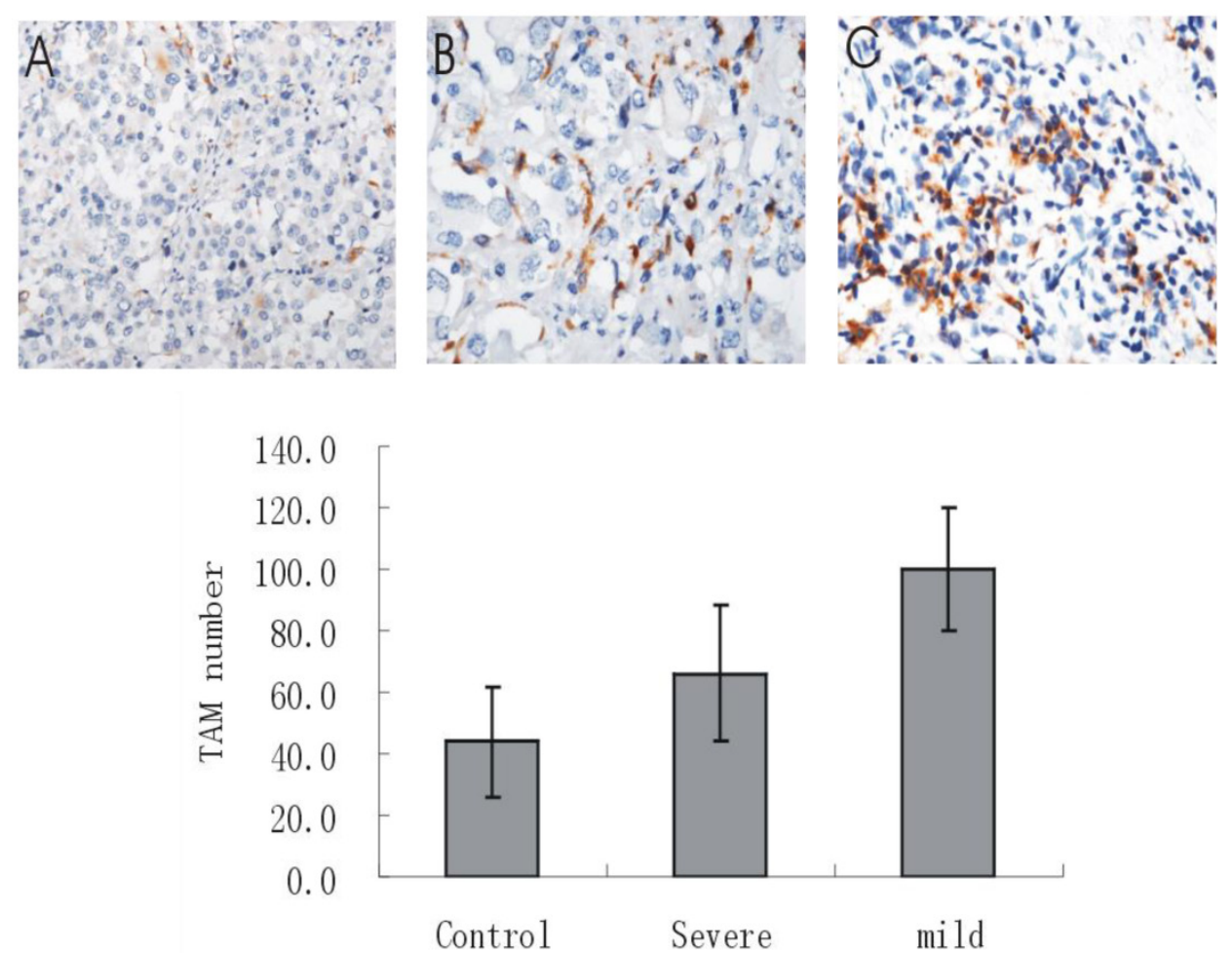

Figure 5. Machrophage infiltration in different ischemia group. Much higher number of TAM could be found in the mild ischemia group (14 days) compared with the control group and severe ischemia group. $* \mathrm{P}<0.05$, compared with the control group. $* * P<0.05$, compared with both the control group and the severe ischemia group. Pictures shown were taken at $200 \times$ magnification. 


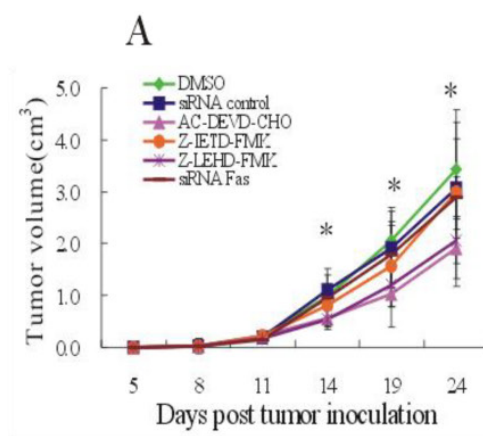

$\mathrm{C}$

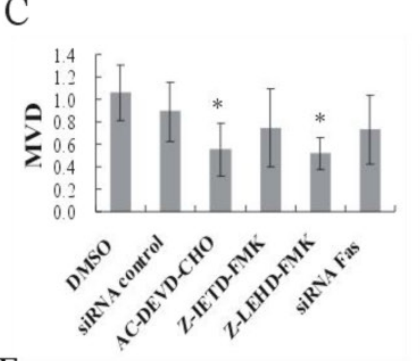

E

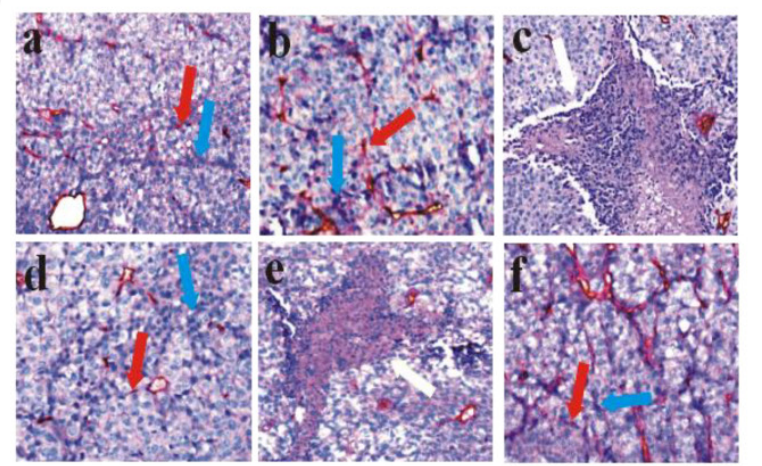

B

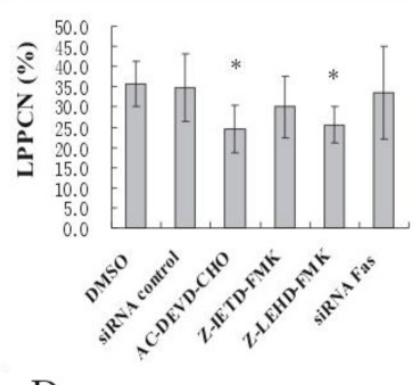

D

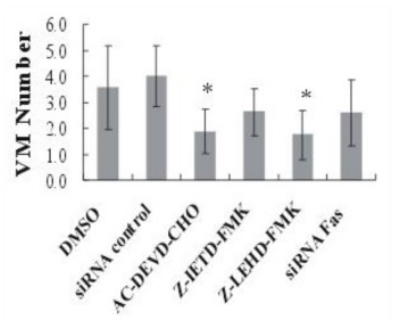

F

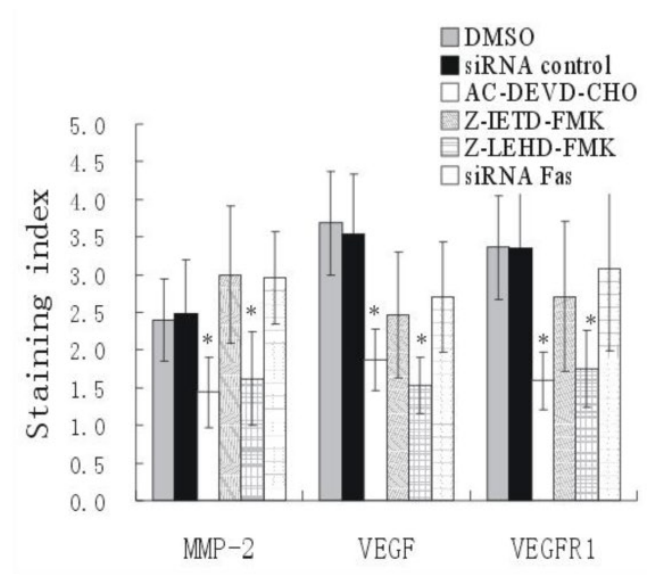

G

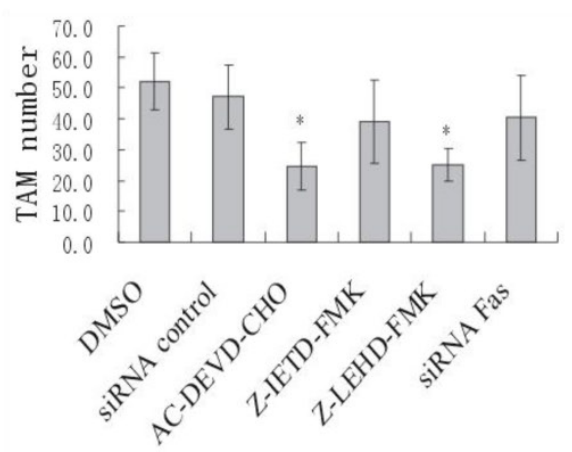

Figure 6. Reduced tumor volume and angiogenesis after caspase inhibition. (A) A plot of tumor volume versus days of tumor growth. (B), (C) and (D) LPPCN number, MVD and VM number were significantly lower after treatment of caspase 3 or caspase 9 inhibitor. (E) Endomucin-PAS staining of tumor tissues in different groups. Representative images showed decreased number of LPPCN, MVD and VM shown in B, C and D as well as larger necrotic areas after caspase3 or caspase9 inhibition. The blue arrow: LPPCN; the red arrow: endomucin-positive microvessels; the white arrow: necrotic areas. (F) and (G) The staining indices of MMP-2, VEGF, VEGFR1 and number of TAM were significantly lower after treatment with caspase3 or caspase9 inhibition. $* P<0.05$, compared to DMSO control groups. Data were shown as mean \pm SEM from five mice per group $(200 \times)$.

\section{Discussion}

Tumor hypoxia is, clinically, an independent prognostic factor for poor patient survival. Hypoxic tumor cells seem to be more aggressive, with reduced apoptosis and increased metastatic potential (22). Hypoxia is also potent driver of a multitude of molecular signalling pathways that allow cells to survive and thrive in the hostile tumor microenvironment and can induce epithelial-mesenchymal transition (EMT) as well as help to drive the linkage between EMT and cancer stem cells (CSCs) (23). Recent study showed that hypoxia clearly has the potential to exert significant effect on the maintenance and evolution of CSCs and hypoxia had been reported to promote the self-renewal capacity of $\mathrm{CD}_{133^{+}}$glioma CSCs and their undifferentiated phenotype through HIF1a activation. Hypoxia could also influence the behavior of CSCs. Neuroblastoma CSCs were preferentially found in hypoxic regions of the tumor and hypoxia significantly increased the tumorigenic fraction of Oct4-expressing side population cells (24).

Tumors with high IFP showed high resistance to blood flow and high fractions of hypoxic cells, whereas both hypoxia and high IFP have been proved to be as biomarkers for metastasis $(25,26)$. Physiological responses of the organism may reduce IFP through induction of orderly cell death. Accumulating evidence indicates that cell death with characteristics of both apoptosis and necrosis can occur (13, 27-29). We previously demonstrated a pattern of cell death with both apoptotic and necrotic morphology and shared the same molecular players with mitochondrial pathway of apoptosis which was named LPPCN and could be observed in a variety of highly invasive malignancies apart from melanoma (17).

Calcium overload triggers mitochondrial damage and calcium-dependent protease activation which 
resulted in both apoptotic and necrotic like types of cell death. Calpain activation which cleaves several essential cytoskeletal proteins (30) and caspase-mediated proteolysis can both play a role in necrosis (31). This study revealed that chronic mild hypoxia induced intracellular calcium influx which resulted in mitochondrial dysfunction, cytochrome c release, and ultimately caspase activation or direct activation of calpains and possibly subsequent LPPCN formation. It was also shown that short or long term of severe ischemia can both induce dramatic elevation of calcium and large areas of necrosis while only long term of mild ischemia can cause mild increase of calcium and formation of LPPCN. It may be that rapid and dramatic increase of cytoplasmic calcium concentration resulted in necrosis by acute activation of great amount of calcium dependent proteases and ensuing rapid, unregulated and complete catastrophic cleavage of structural proteins while mild elevation of calcium may activate an orderly and programmed cascade and regulated degradation of cell components and displayed both features of apoptosis and necrosis.

High focal microphage infiltration into tumors where was poorly vascularized and hypoxic is associated with increased tumor angiogenesis and poor prognosis due to production of angiogenic factors like VEGF and TNFa (32). In this study, higher microphage infiltration and VEGF expression can be found in the tumors with LPPCN and especially in the LPPCN areas. Thus, we presumed that chronic mild hypoxia induced LPPCN may recruit the infiltration of macrophages into the LPPCN areas and then VEGF was released to promote angiogenesis locally.

To extend our above mentioned observation, a B16 melanoma-bearing animal model in which LPPCN formation was inhibited with caspase inhibitors was established. LPPCN inhibition restrained the number of microvessels and VM, and resulted in large necrotic areas in the tumor and slowed tumor growth. We presumed that LPPCN may be a general mechanism to eliminate tumor cells that cannot bear the chronic hypoxic environments during rapid tumor growth which reduced the high IFP and promote angiogenesis to ensure the survival of most other cells. It was reported that inhibition of programmed cell death impairs endothelium dependent angiogenesis as apoptosis of endothelial cells is needed in the formation of new vascular tissue (33). Our results demonstrated that not only angiogenesis in normal tissues requires cell death for removing of superfluous cells but also the tumor angiogenesis need some tumor cells to die to provide a spatial architecture for capillary formation by both endothelial cells and tumor cells. HIF-1ais a endogenous marker of tumor hypoxia and a dramatic rise of the expression of
HIF-1awas found at $0.5 \% \mathrm{O}_{2}$ tension while a drop at $0 \%$ due to an effect of anoxia on overall transcription and translocation(34). In this study, high levels of HIF-1 $\alpha$ and pimonidazole were found in LPPCN and peri-LPPCN areas suggesting these regions were relatively hypoxic. Little expression of HIF-1acan be detected in LPPCN cells which may be attributed to extreme hypoxia and the overall follow up transcription changes. Under hypoxia, HIF-1atranslocates to the nucleus and binds to hypoxia-responsive elements of hypoxia-regulated genes including $\operatorname{VEGF(35),~}$ VEGFR1(36) and MT1-MMP which is a potent activator of MMP-2(37). VEGF has been demonstrated to be a major contributor to angiogenesis, both increasing the number of capillaries $(38,39)$ and played an important role in VM formation (40). MMP-2 is required for VM formation by degradation of Laminin5 to promote the motility of aggressive tumor cells. The tumor cells lining the VM tube express endothelial cell-associated genes such as vascular endothelial-cadherin (VE-Cadherin). The mechanisms underlying VM formation may be explained by the hypoxia conditions in which the tumor cells have great flexibility in altering their phenotype to mimic the behavior of endothelia $(4,41-43)$. Our results of higher expression of HIF-1a, VEGF, VEGFR1, MMP-2 and VE-Cadherin in the peri-LPPCN regions revealed that hypoxia induced a more aggressive phenotype of these cells via HIF-1a to promote angiogenesis (recruitment of blood vessels and higher plasticity to mimic the vascular formation) and alleviate hypoxia conditions. The administration of caspase 3 or caspase9 inhibitor down-regulated the expression of MMP-2, VEGF and VEGFR1 in tumor cells while no significant changes were observed as to the expression of HIF-1aand VE-Cadherin. The possible reason may be that inhibition of caspase3 or caspase9 resulted in inability of LPPCN formation and subsequent impairment of microvessels and VM formation which lead to even more seriously hypoxia conditions and thus large areas of necrosis. As mentioned above, expression of HIF-1a cannot be found in extremely hypoxia conditions which resulted in decrease of expression of MMP-2, VEGF and VEGFR1. The reason why no significances were found as to the expression of VE-Cadherin in different treatment groups is still puzzling and further study is still required.

In conclusion, we described a phenomenon wherein a hypoxic environment and tumor growth induced tumor cell death which had both apoptotic and necrotic characteristics and may provide needed space for tumor angiogenesis to maintain continuous tumor growth. New molecular targets for therapeutic tumor angiogenesis intervention may also be developed based on our findings. The exact molecular 
events of LPPCN and differences and relationship with apoptosis and necrosis in aspects of signal transduction and reasons of their different responses to hypoxia are still needed to be elaborated further.

\section{Supplementary Material}

Supplementary methods, supplementary table and figure. http://www.jcancer.org/v07p0022s1.pdf

\section{Acknowledgement}

This work was supported by a grant from a Key Project of the National Natural Science Foundation of China (No. 81230050), Project of the National Natural Science Foundation of China (No.81172046, No.81173091, No. 81101724, No. 81201791), Key project of the Tianjin Natural Science Foundation (No. 12JCZDJC23600), the Tianjin Natural Science Foundation (No. 12JCYBJC15500).

\section{Competing Interests}

The authors have declared that no competing interest exists.

\section{References}

1. Frena A, Mazziotti A, Zanetti G, Gozzetti G. An unusual case of hepatic tumor. HPB Surg. 1991; 3: 297-300.

2. Zhang S, Zhang D, Wang Y, Zhao W, Guo H, Zhao X, Sun B. Morphologic research of microcirculation patterns in human and animal melanoma. Med Oncol. 2006; 23: 403-409.

3. Chang YS, di Tomaso E, McDonald DM, Jones R, Jain RK, Munn LL. Mosaic blood vessels in tumors: frequency of cancer cells in contact with flowing blood. Proc Natl Acad Sci U S A. 2000; 97: 14608-14613.

4. Folberg R, Hendrix MJ, Maniotis AJ. Vasculogenic mimicry and tumor angiogenesis. Am J Pathol. 2000;156: 361-381.

5. Paulis YW, Soetekouw PM, Verheul HM, Tjan-Heijnen VC, Griffioen AW. Signalling pathways in vasculogenic mimicry. Biochim Biophys Acta. 2010; 1806: 18-28.

6. Maniotis AJ, Folberg R, Hess A, Seftor EA, Gardner LM, Pe'er J, Trent JM, Meltzer PS, Hendrix MJ. Vascular channel formation by human melanoma cells in vivo and in vitro: vasculogenic mimicry. Am J Pathol. 1999; 155: 739-752.

7. Gulliksrud K, Galappathi K, Rofstad EK. Interstitial Fluid Pressure and Vascularity of Intradermal and Intramuscular Human Tumor Xenografts. Int J Radiat Oncol Biol Phys. 2011; 80:258-64.

8. Cairns R, Papandreou I, Denko N. Overcoming physiologic barriers to cancer treatment by molecularly targeting the tumor microenvironment. Mol Cancer Res. 2006 ; 4: 61-70.

9. Griffon-Etienne G, Boucher Y, Brekken C, Suit HD, Jain RK. Taxane-induced apoptosis decompresses blood vessels and lowers interstitial fluid pressure in solid tumors: clinical implications. Cancer Res. 1999; 59: 3776-3782.

10. Borschmann K, Moore K, Russell M, Ledgerwood K, Renehan E, Lin X, Brown C, Sison J. Overcoming barriers to physical activity among culturally and linguistically diverse older adults: a randomised controlled trial. Australas J Ageing. 2010; 29: 77-80.

11. Vartanian AA, Burova OS, Stepanova EV, Baryshnikov AY. The involvement of apoptosis in melanoma vasculogenic mimicry. Melanoma Res. 2007; 17: 1-8.

12. Hitomi J, Christofferson DE, Ng A, Yao J, Degterev A, Xavier RJ, Yuan J. Identification of a molecular signaling network that regulates a cellular necrotic cell death pathway. Cell. 2008; 135: 1311-1323.

13. Galluzzi L, Kroemer G. Necroptosis: a specialized pathway of programmed necrosis. Cell. 2008; 135: 1161-1163.

14. Wrighton KH. Cell death: A killer puts a stop on necroptosis. Nat Rev Mol Cell Biol. 2011; 12:279.

15. Vandenabeele P, Galluzzi L, Vanden Berghe T, Kroemer G. Molecular mechanisms of necroptosis: an ordered cellular explosion. Nat Rev Mol Cell Biol. 2010; 11: 700-714.

16. Sun B, Zhang D, Zhang S, Zhang W, Guo H, Zhao X. Hypoxia influences vasculogenic mimicry channel formation and tumor invasion-related protein expression in melanoma. Cancer Lett. 2007; 249: 188-197.
17. Zhang S, Li M, Zhang D, Xu S, Wang X, Liu Z, Zhao X, Sun B. Hypoxia influences linearly patterned programmed cell necrosis and tumor blood supply patterns formation in melanoma. Lab Invest. 2009; 89: 575-586.

18. Goto T1, Fukuyama N, Aki A, Kanabuchi K, Kimura K, Taira H, Tanaka E, Wakana N, Mori H, Inoue H. Search for appropriate experimental methods to create stable hind-limb ischemia in mouse. Tokai J Exp Clin Med. 2006; 31: 128-132.

19. Sun B1, Qie S, Zhang S, Sun T, Zhao X, Gao S, Ni C, Wang X, Liu Y, Zhang L. Role and mechanism of vasculogenic mimicry in gastrointestinal stromal tumors. Hum Pathol. 2008; 39: 444-451.

20. Weidner N. Tumour vascularity and proliferation: clear evidence of a close relationship. J Pathol. 1999; 189: 297-299.

21. Shirinifard A1, Gens JS, Zaitlen BL, Popławski NJ, Swat M, Glazier JA. 3D multi-cell simulation of tumor growth and angiogenesis. PLoS One. 2009; 4: e7190.

22. Yun Z, Lin Q. Hypoxia and regulation of cancer cell stemness. Adv Exp Med Biol. 2014:772:41-53.

23. Philip B, Ito K, Moreno-Sánchez R, Ralph SJ. HIF expression and the role of hypoxic microenvironments within primary tumours as protective sites driving cancer stem cell renewal and metastatic progression. Carcinogenesis. 2013; 34:1699-707.

24. Marie-Egyptienne DT1, Lohse I, Hill RP. Cancer stem cells, the epithelial to mesenchymal transition (EMT) and radioresistance: potential role of hypoxia. Cancer Lett. 2013; 341:63-72.

25. Raju B, Haug SR, Ibrahim SO, Heyeraas KJ. High interstitial fluid pressure in rat tongue cancer is related to increased lymph vessel area, tumor size, invasiveness and decreased body weight. J Oral Pathol Med. 2008; 37: 137-144.

26. Tannock IF. Tumor physiology and drug resistance. Cancer Metastasis Rev. 2001; 20: 123-132.

27. Rosenbaum DM, Degterev A, David J, Rosenbaum PS, Roth S, Grotta JC, Cuny GD, Yuan J, Savitz SI. Necroptosis, a novel form of caspase-independent cell death, contributes to neuronal damage in a retinal ischemia-reperfusion injury model. J Neurosci Res. 2010; 88: 1569-1576.

28. Ch'en IL, Tsau JS, Molkentin JD, Komatsu M, Hedrick SM. Mechanisms of necroptosis in T cells. J Exp Med. 2011; 208:633-41.

29. Chen BN, Du GH. Necroptosis: a new mechanism of cell death. Sheng Li Ke Xue Jin Zhan. 2010; 41: 95-99.

30. Bozym RA, Patel K, White C, Cheung KH, Bergelson JM, Morosky SA, Coyne $\mathrm{CB}$. Calcium signals and calpain-dependent necrosis are essential for release of coxsackievirus B from polarized intestinal epithelial cells. Mol Biol Cell. 2011; 22: 3010-3021.

31. Edelstein CL, Shi Y, Schrier RW. Role of caspases in hypoxia-induced necrosis of rat renal proximal tubules. J Am Soc Nephrol. 1999; 10: 1940-1949.

32. Leek RD, Landers RJ, Harris AL, Lewis CE. Necrosis correlates with high vascular density and focal macrophage infiltration in invasive carcinoma of the breast. Br J Cancer. 1999; 79: 991-995.

33. Segura I, Serrano A, De Buitrago GG, González MA, Abad JL, Clavería C, Gómez L, Bernad A, Martínez-A C, Riese HH. Inhibition of programmed cell death impairs in vitro vascular-like structure formation and reduces in vivo angiogenesis. FASEB J. 2002; 16: 833-841.

34. Jiang BH, Semenza GL, Bauer C, Marti HH. Hypoxia-inducible factor 1 levels vary exponentially over a physiologically relevant range of $\mathrm{O} 2$ tension. Am J Physiol. 1996; 271: C1172-1180.

35. Koukourakis MI1, Giatromanolaki A, Skarlatos J, Corti L, Blandamura S, Piazza M, Gatter KC, Harris AL. Hypoxia inducible factor (HIF-1a and HIF-2a) expression in early esophageal cancer and response to photodynamic therapy and radiotherapy. Cancer Res. 2001. 61: 1830-1832.

36. Harris AL. Hypoxia--a key regulatory factor in tumour growth. Nat Rev Cancer. 2002: 2: 38-47.

37. Munoz-Najar UM, Neurath KM, Vumbaca F, Claffey KP. Hypoxia stimulates breast carcinoma cell invasion through MT1-MMP and MMP-2 activation. Oncogene. 2006; 25: 2379-2392.

38. Goodsell DS. The molecular perspective: VEGF and angiogenesis. Stem Cells. 2003; 21: 118-119.

39. Goodsell DS. The molecular perspective: VEGF and angiogenesis. Oncologist. 2002; 7: 569-570.

40. Wang JY, Sun T, Zhao XL, Zhang SW, Zhang DF, Gu Q, Wang XH, Zhao N, Qie S, Sun BC. Functional significance of VEGF-a in human ovarian carcinoma: role in vasculogenic mimicry. Cancer Biol Ther. 2008; 7: 758-766.

41. Ruf W1, Seftor EA, Petrovan RJ, Weiss RM, Gruman LM, Margaryan NV, Seftor RE, Miyagi Y, Hendrix MJ. Differential role of tissue factor pathway inhibitors 1 and 2 in melanoma vasculogenic mimicry. Cancer Res. 2003; 63: 5381-5389.

42. Folberg R, Maniotis AJ. Vasculogenic mimicry. APMIS. 2004; 112: 508-525.

43. Vanden Berghe T1, Vanlangenakker N, Parthoens E, Deckers W, Devos M, Festjens N, Guerin CJ, Brunk UT, Declercq W, Vandenabeele P. Necroptosis, necrosis and secondary necrosis converge on similar cellular disintegration features. Cell Death Differ. 2010; 17: 922-930. 